\title{
Estenose bilateral de seio venoso transverso associado a papiledema - Relato de caso
}

\author{
Bilateral transverse sinus stenosis associated with papilledema - Case Report
}

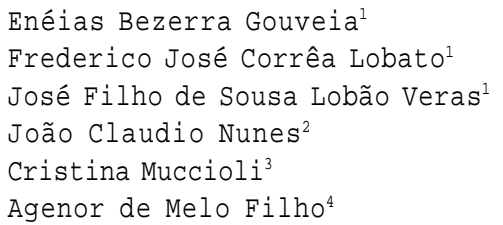

\section{R E S U M O}

Relatar um caso de estenose bilateral de seio venoso transverso cursando com papiledema e discutir evolução clínica, alterações fundoscópicas, diagnóstico diferencial com pseudotumor cerebral e descrever nova proposta terapêutica (por meio do uso de "stent"). Relato de Caso: Paciente de 39 anos, do sexo feminino, portador de estenose bilateral de seio transverso associada a papiledema, apresentando baixa de acuidade visual, restrição de campos visuais e cefaléia. O diagnóstico foi confirmado por meio de angiografia cerebral. Instituiu-se terapêutica intervencionista, por meio do implante de "stent" via angiográfica cerebral, com remissão dos sinais e sintomas aos exames oftalmológico e neurológico. Discussão: Neste trabalho descrevemos os achados radiológicos e oftalmológicos da estenose bilateral do seio transverso e apresentamos nova terapêutica por meio de implante de "stent" via angiografia cerebral, com melhora documentada dos níveis pressóricos intracranianos, dos achados campimétricos visuais e dados do exame oftalmológico após o procedimento. Não há relato na literatura internacional de estenose bilateral do seio venoso associado a papiledema. Por se tratar do primeiro relato na literatura, os achados descritos sugerem fortemente que esta associação possa ser parte de uma nova entidade nosológica.

Descritores:Constriçãopatológica/radiografia;Angiografiacerebral;Pseudotumorcerebral; Papiledema;Transtomosdavisão/etiologia;Relatodecaso;Feminino;Adulto

I N T ROD U Ç ̃̃ O

Pseudotumor cerebral (PTC) ou hipertensão intracraniana idiopática (HII) é uma doença ameaçadora que compromete a visão, afetando tipicamente mulheres obesas em idade fértil ${ }^{(1-3)}$. A incidência é oito vezes maior nas mulheres. Pacientes atípicos para pseudotumor cerebral, incluindo mulheres magras, homens e crianças, devem ser avaliados à procura de causa subjacente. A ressonância magnética com contraste e ressonância magnética com venografia é recomendada nesses $\operatorname{casos}^{(4)}$.

Pseudotumor cerebral é uma síndrome de pressão intracraniana aumentada que ocorre sem uma lesão anatômica estrutural demonstrada. Os critérios clínicos que demandam procura pela causa primária, incluem: (1) o paciente deve estar alerta e acordado, (2) não deverá haver nenhum sinal de lateralização ao exame neurológico além da paralisia do músculo reto lateral, (3) o papiledema deverá ser causado por aumento da pressão intracraniana e não pelo pseudopapiledema (4) o estudo de neuroimagem deverá ser normal, e (5) a punção lombar demonstra aumentada pressão liquórica, concentração protéica normal ou baixa, glicose normal e contagem celular 
normal. Quando nenhuma causa subjacente é encontrada, o distúrbio é denominado hipertensão intracraniana idiopática ${ }^{(4)}$.

Os sinais e sintomas neurológicos são relacionados à hipertensão venosa passiva ou congestão como resultado do aumento pressórico no seio sagital superior ou trombose do seio venoso. Os achados clínicos são determinados por volume, direção e taxa de drenagem venosa ${ }^{(5)}$.

Os sinais e sintomas exceto por "tinnitus" pulsátil, variam e dependem aparentemente da diminuição da drenagem venosa, que pode determinar, aumento da pressão intracraniana, papiledema e déficit neurológico transitório ou persistente ${ }^{(6)}$. As lesões oclusivas que ocorrem nos seios durais produzem trombose parcial e estenose dos mesmos e podem se manifestar com déficits neurológicos distantes da lesão. Estes déficits podem incluir aumento da pressão intracraniana, deterioração mental, hemorragia, e déficits focais $\operatorname{remotos}^{(7)}$. Alterações neuroftalmológicas incluem a presença de papiledema, paralisia dos nervos cranianos, pulso sincrônico abrupto, "tinnitus" (raramente) e proptose, que pode ser bilateral ${ }^{(5)}$.

Quase todos os pacientes com pseudotumor cerebral têm cefaléia, apesar do papiledema ser detectado ocasionalmente quando em exame oftalmológico de rotina. A cefaléia freqüentemente é diária, retro-ocular e piora com movimentos oculares ${ }^{(8)}$.

Apesar de haver associação entre papiledema de grau alto e a perda visual ${ }^{(9)}$, a aparência do nervo óptico não prediz o resultado visual do paciente ${ }^{(10)}$.

O teste mais útil na avaliação da função visual em pacientes com pseudotumor cerebral é a perimetria ${ }^{(11)}$. As anormalidades mais freqüentes do campo visual são o aumento da mancha cega, constrição de campo generalizada e defeitos campimétricos nasais ${ }^{(10)}$.

O tratamento não deve ser baseado apenas na aparência do nervo óptico ${ }^{(4)}$. A terapia tradicional emprega diuréticos, particularmente inibidores da anidrase carbônica. A anidrase carbônica, presente no plexo coroidal, tem papel principal na secreção liquórica. Um estudo demonstrou que a acetazolamida foi efetiva em $75 \%$ dos pacientes com pseudotumor cerebral ${ }^{(12)}$.

A importância da redução do peso no tratamento do PTC foi avaliada permanecendo uma modalidade de grande valor no tratamento da $\mathrm{HII}^{(13-14)}$.

O papel da cirurgia lomboperitoneal no tratamento cirúrgico do PTC foi examinado concluindo-se que a derivação lomboperitoneal é uma alternativa segura e eficaz para pacientes com PTC quando a cefaléia e a perda visual são refratárias ao tratamento medicamentoso ${ }^{(15)}$.

O advento da ressonância magnética propiciou nova abordagem para a hipertensão intracraniana idiopática através da demonstração de lesões antes não evidenciadas por outros métodos diagnósticos de imagem. Assim, distinguiram-se outras lesões como causadores de hipertensão intracraniana do grupo antes englobado pela entidade pseudotumor cerebral.

Neste estudo, os autores apresentam um caso de estenose bilateral de seio venoso transverso cursando com papiledema e discutem a evolução clínica, as alterações fundoscópicas, diagnóstico diferencial com pseudotumor cerebral e a proposta terapêutica. Relata-se um caso de estenose bilateral de seio venoso transverso causando síndrome que se assemelha a pseudotumor cerebral. Não se encontram relatos na literatura entre estenose bilateral do seio transverso e papiledema. Consideramos que estas informações evidenciam o fato de que podemos estar diante de nova entidade nosológica, outrora classificada como pseudotumor cerebral.

RELATO DE CASO

V.G.S., do sexo feminino, leucoderma, 39 anos de idade, costureira, procedente da cidade de São Paulo, apresentou quadro de cefaléia fronto-occipital bilateral, de forte intensidade, tipo compressiva, constante, com períodos de piora e melhora espontânea, havendo exacerbação da dor aos esforços, após almoço e posição supina, e alívio relativo com analgésicos, associada à foto e fonofobia, seguida, após cinco dias, de episódio agudo de baixa de acuidade visual em ambos os olhos, acompanhados de otorragia em ouvido esquerdo. Os antecedentes mórbidos pessoais e familiares foram irrelevantes.

Ao exame oftalmológico, a paciente apresentava acuidade visual com a correção de 20/60 em OD e 20/100 em OE. Os teste de placas pseudoisoquiasmáticas não evidenciava alteração. Reflexo fotomotor e consensual era normal em ambos os olhos. A biomicroscopia em ambos os olhos foi compatível com a normalidade. Os movimentos oculares extrínsecos estavam preservados. A tonometria de aplanação apresentou pressão intra-ocular de $9 \mathrm{mmHg}$ no olho direito e de $10 \mathrm{mmHg}$ no olho esquerdo. À fundoscopia observou-se presença de protusão, hiperemia e edema papilar bilateral com borramento das bordas (figura 1a e 1b).

A angiofluoresceinografia demonstra hiperfluorescência transmitida peripapilar e hiperfluorescência por vazamento tardio nas bordas papilares compatível com papiledema bilateral (figura 1c).

Campimetria computadorizada de Humphrey, com programa Central 30-2 "Threshold test", utilizando estímulo "White II", evidenciou diminuição acentuada de sensibilidade na periferia dos quatro quadrantes em ambos os olhos (figura 2). A análise do líquor, exames laboratoriais e sorológicos foram compatíveis com a normalidade.

A tomografia computadorizada (TC) e a ressonância magnética (RM) do encéfalo apresentaram-se sem alterações significativas, demonstrando sinais de hipertensão intracraniana e provável estenose bilateral dos seios transversos. A angiografia cerebral confirmou estenose grave na transição do terço médio com o distal de ambos os seios transversos, havendo comprometimento luminar de aproximadamente 50\% (figura 3). No cateterismo venoso, a pressão do golfo jugular, foi de $9 \mathrm{mmHg}$ à direita e $8 \mathrm{mmHg}$, na porção inicial dos seios transversos foi $28 \mathrm{mmHg}$ à direita e $31 \mathrm{mmHg}$, na porção distal do seio sagital superior de $45 \mathrm{mmHg}$.

A paciente foi medicada com prednisona, via oral, $20 \mathrm{mg}$ ao dia e acetazolamida oral, $750 \mathrm{mg} / \mathrm{dia}$, sendo descontinuado o uso desta devido os efeitos colaterais (parestesia nos pés, náuseas e irritação gástrica). 


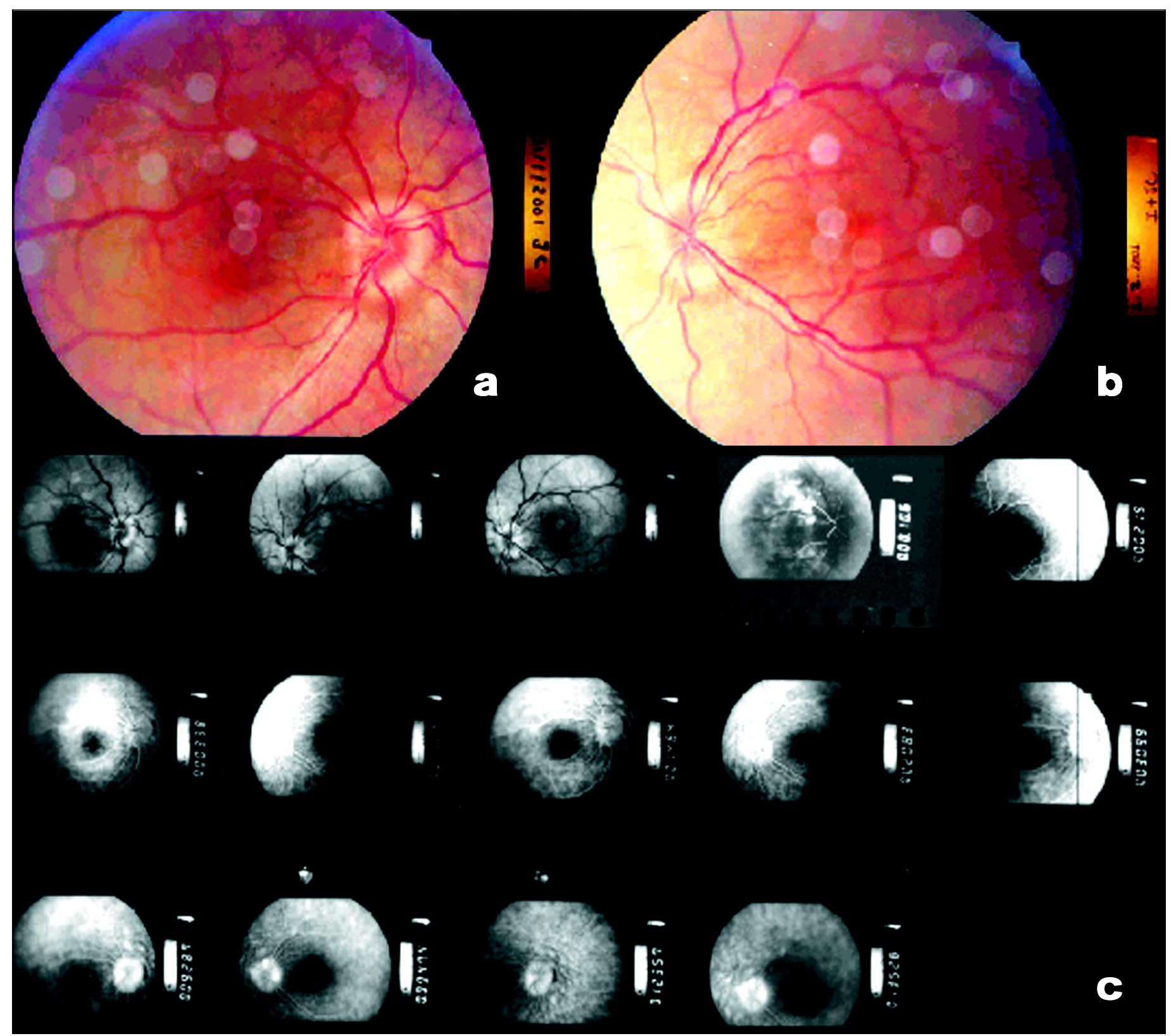

Figura1-Fundoscoplaporocasiãododiagnósticodoolhodireito(a)eesquerdo(b).Fotocampomédiodemonstrandoseqüênciaangiofluoresceinográfica emambos os olhos porocasiãododiagnóstico(c)

A paciente foi submetida a implante de "stent" via angiográfica do tipo "Velocity" de 4,5 mm de diâmetro x $20 \mathrm{~mm}$ de comprimento (figura 3 ), evoluindo com melhora significativa do quadro de cefaléia, restauração dos níveis pressóricos à normalidade (pressão média de $7 \mathrm{mmHg}$ no seio sagital superior, $6 \mathrm{mmHg}$ no seio transverso esquerdo e $5 \mathrm{mmHg}$ no seio sigmóide esquerdo) após o procedimento (figura 3 ). Houve melhora da campimetria computadorizada (figura 2) e da acuidade visual (20/30 em ambos os olhos) e o controle angiográfico demonstrou regressão importante da estenose com boa luz ao nível do "stent" após dois meses do procedimento intervencionista. Neste mesmo período, houve diminuição da hiperemia e do edema de papila, sendo observada maior nitidez das bordas papilares, à fundoscopia, que se mantiveram nos exames subseqüentes após quatro meses da intervenção radiológica, apresentando acuidade visual corrigida de 20/25 em ambos os olhos, não constando alterações campimétricas e os níveis pressóricos estavam compatíveis com a normalidade.

\section{I S C US S ÃO}

A hipótese inicial da paciente foi pseudotumor cerebral, que é caracterizada por aumento da pressão intracraniana, sem evidência de lesão expansiva intracraniana, exame de imagem 


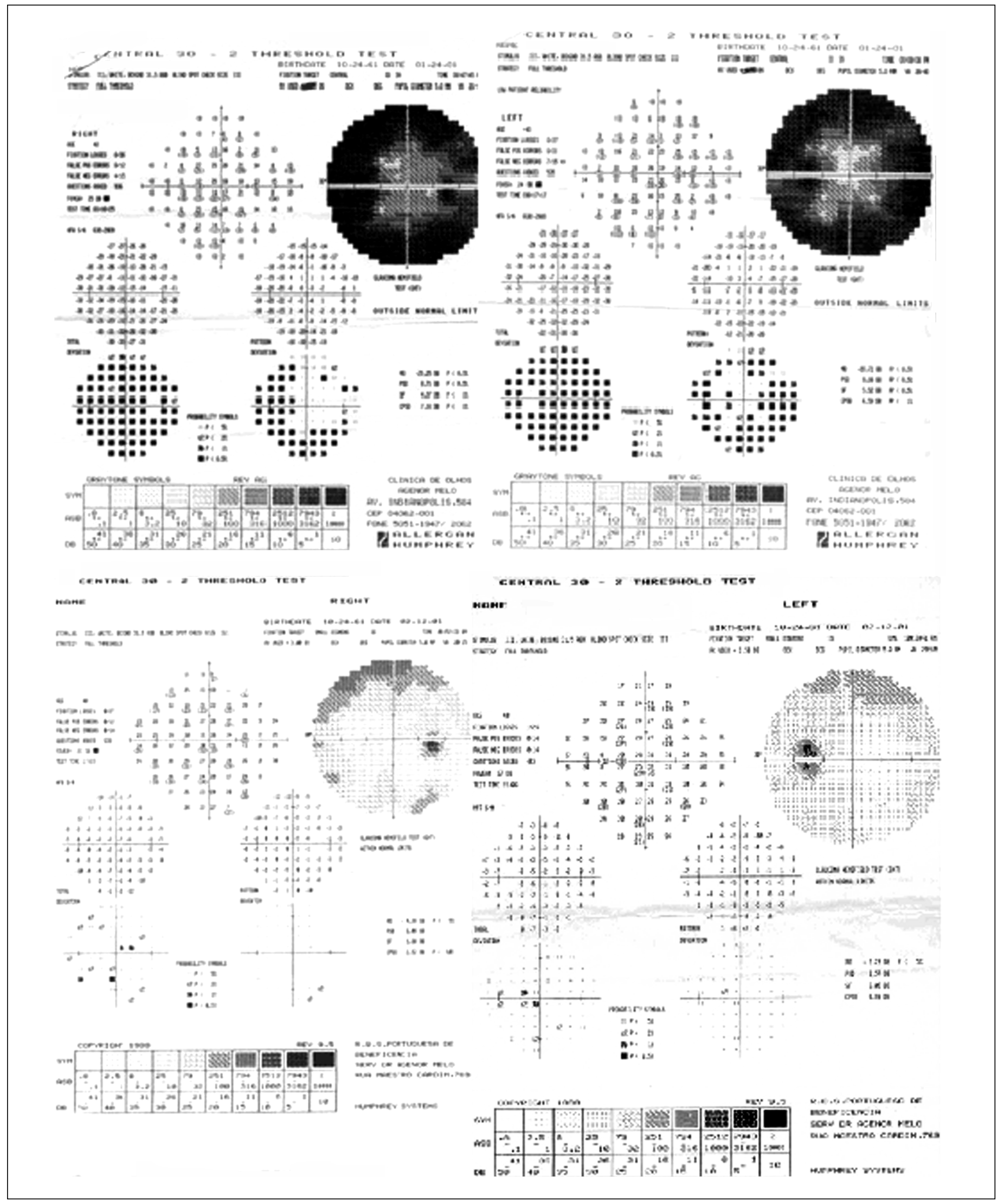

Figura 2-Campimetriacomputadorizada (Central30-2 “TresholdTest”-Humphreylnst.)porocasiãododiagnóstico(superiordireitaeesquerda)e após intervençãocinúrgica (inferiordireitaeesquerda) 

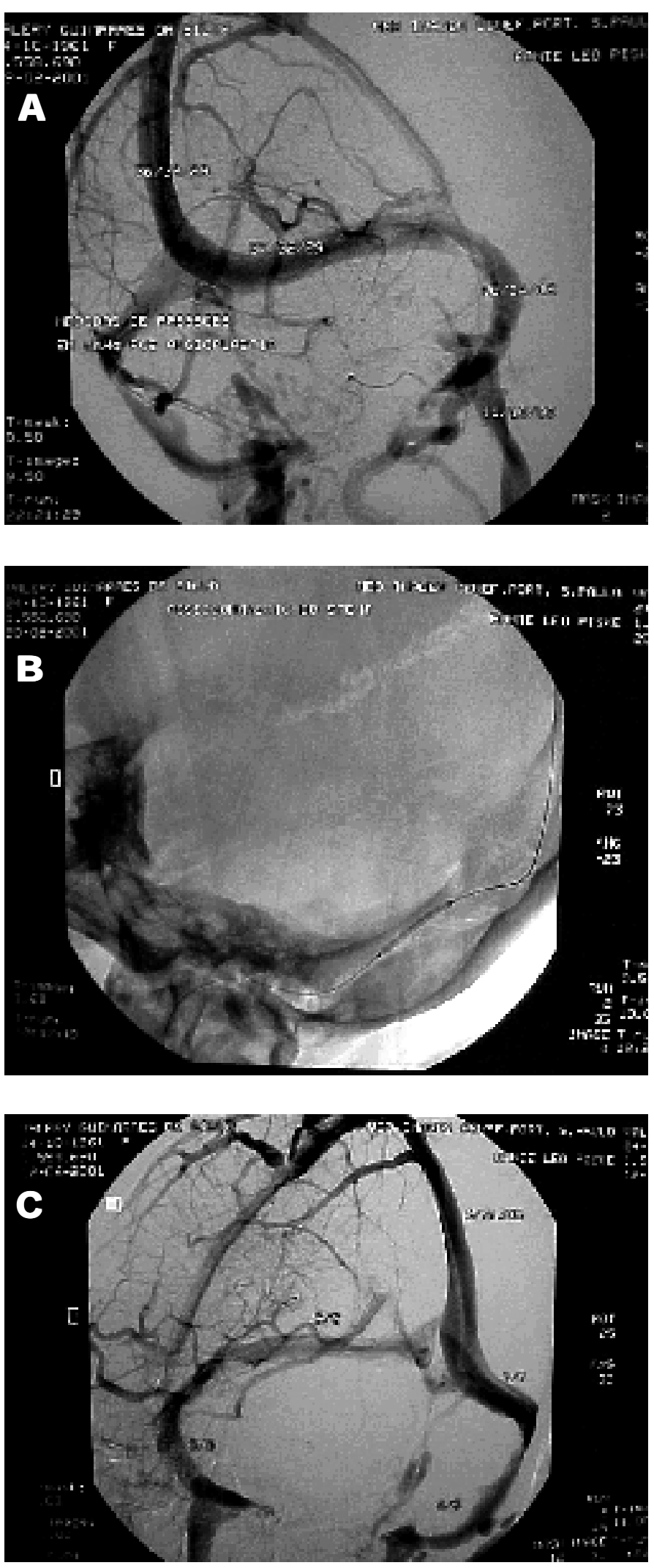

Figura 3-Angiografia cerebral demonstrandoa estenose bilateral de seiovenoso transverso e níveis pressóricos elevados pré-estenose (fotografia A), colocação do "stent"via angiográfica no seio venoso transverso (fotografia B) enovocontroleangiográfico pós-procedimento comníveis pressóricos do liquor diminuídos (fotografia C) normal e líquor cefalorraquidiano (LCR) normal, ocorrendo mais comumente em mulheres obesas em sua segunda a quarta década de $v_{i d a}{ }^{(16)}$. Uma vez que se tratava de uma paciente magra (forma atípica), de meia idade, com quadro de cefaléia e com episódio agudo de baixa acuidade visual e apresentando à fundoscopia papiledema bilateral, se tornava indispensável investigação mais apurada para afastamento de etiologias outras que pudessem estar gerando o quadro, já que o diagnóstico de PTC é diagnóstico de exclusão, sendo à TC com contraste, a RNM e em alguns casos a angiografia cerebral indispensáveis em casos de suspeita de PTC com manifestações atípicas.

A paciente não apresentava alterações significativas à TC e à RM, demonstrando sinais de hipertensão intracraniana e LCR normal e um curso clínico progressivo. Para esclarecer este fato a paciente foi submetida ao exame de angiografia cerebral que afastou a possibilidade de má formação arteriovenosa, de trombose parcial do seio dural e de PTC por confirmar um diagnóstico etiológico - estenose de seio transverso, com comprometimento luminar de aproximadamente $50 \%$, o que podemos supor que seria esta alteração a determinadora do aumento da pressão intracraniana, com o subseqüente desenvolvimento de papiledema e o prejuízo visual.

Como o quadro era bastante sugestivo inicialmente de PTC, a paciente teria sido tratada com prednisona, via oral, $20 \mathrm{mg}$ ao dia e acetazolamina oral $750 \mathrm{mg} / \mathrm{dia}$, não apresentando resultados satisfatórios uma vez que não se tratou a causa base. A paciente foi examinada pela neurologia que propôs a implantação de "stent" via angiográfica, no seio venoso transverso, que permitiria a ampliação da luz venosa e assim uma redução nos níveis pressórios intracraniano.

No caso apresentado, os autores propuseram o diagnóstico de estenose de seio transverso bilateral, pelo fato de a paciente apresentar quadro de hipertensão intracraniana com cefaléia intensa e papiledema bilateral e angiografia cerebral compatível com estenose bilateral de seio venoso transverso. A respeito das opções terapêuticas, não há um tratamento clínico comprovado, tendo sido proposto o implante de "stent" via angiográfica no seio venoso transverso para correção da estenose, com melhora documentada do campo visual e da fundoscopia, e melhora dos sintomas.

Não há relato na literatura da associação de estenose bilateral do seio transverso a papiledema. Cumpre considerar que o presente relato só foi possível mediante os avanços diagnóstico de imagem, proporcionando hipótese diagnóstica mais precisa. $\mathrm{O}$ quadro descrito é sugestivo de pseudotumor cerebral que só foi afastado depois de uma investigação mais profunda, assim a definição de sinais e sintomas desta doença, formas diagnósticas, modalidade de tratamento, diagnóstico diferencial e prognóstico da mesma só acontecerá quando outros pacientes forem estudados.

\section{A B S T R A C T}

The authors report the case of a female patient, 39 years old, presenting bilateral transverse venous sinus stenosis, associated with papilledema, visual acuity deficit and 
headache. Cerebral angiographic finding of bilateral transverse venous sinus stenosis elucidated the diagnosis. The patient was treated with cerebral angiographic stent implant, with consequent remission of ophthalmologic and neurological clinical findings.

Keywords: Constriction pathologic/radiography; Cerebral angiography; Pseudotumor cerebri; Papilledema; Vision disorders/etiology; Case report; Female; Adult

\section{REFERENCIAS}

1. Brazis PW, Lee AG: Elevated intracranial pressure and pseudotumor cerebri. Curr Opin Ophthalmol 1998;9:27-32.

2. Radhakrishnan K, Thacker AK, Bohlaga NH, Maloo JC; Gerryo SE. Epidemiology of idiopathic intracranial hypertension: a prospective and case-control study. J Neurol Sci 1993;116:18-28.

3. Movsas TZ, Liu GT, Galetta SL, Balcer LJ, Volpe NJ. Current neuroophthalmic therapies. Neurol Clin 2001;19:145-72, vii.

4. Friedman, DI. Papilledema and pseudotumor cerebri. Ophthalmol Clin North Am 2001;14:129-47, ix.

5. Troost BT, Glaser JS. Aneurysms, arteriovenous communications, and related vascular malformations. In: Tasman W, Jaeger EA, editors. Duane's clinical ophthalmology. Philadelphia: JB Lippincott; 1994. v.2. p.13-16.
6. Houser OW, Campbell JK, Campbell RJ, Sundt TM. Arteriovenous malformation affecting the transverse dural venous sinus - an acquired lesion. Mayo Clin Proc 1979;54:651-61.

7. Youmans J. R. Youmans neurological surgery. 4th ed. Philadelpia:WB Saunders; 1997. v. 2.

8. Giuseffi V, Wall M, Siegel PZ, Rojas PB. Symptoms and disease associations in idiopathic intracranial hypertension (pseudotumor cerebri): a case-control study. Neurology 1991;41:239-44.

9. Sergott RC, Savino PJ, Bosley TM. Modified optic nerve sheath decompression provides long-term visual improvement for pseudotumor cerebri. Arch Ophthalmol 1988;106:1384-90.

10. Wall M, George D: Visual loss in pseudotumor cerebri. Incidence and defects related to visual field strategy. Arch Neurol 1987;44:170-5.

11. Rowe FJ, Sarkies NJ: Visual outcome in a prospective study of idiopathic intracranial hypertension. Arch Ophthalmol 1999;117:1571.

12. Tomsak RL, Niffenegger AS, Remler BF: Treatment of pseudotumor cerebri with Diamox (acetazolamide). J Clin Neuro-Ophthalmol 1988;8:93-8.

13. Kupersmith MJ, Gamell L, Turbin R, Peck V, Spiegel P, Wall M. Effects of weight loss on the course of idiopathic intracranial hypertension in women. Neurology 1998;50:1094-8.

14. Johnson LN, Krohel GB, Madsen RW, March GA. The role of weight loss and acetazolamide in the tratament of idiopathic intracranial hypertension (pseudotumor cerebri). Ophthalmology 1998;105:2313-7.

15. Burgett RA, Purvin VA, Kawasaki A. Lumboperitoneal shunting for pseudotumor cerebri. Neurology 1997;49:734-9.

16. Lana-Peixoto MA Neuro-oftalmologia. Sistema sensorial - parte II.Revisão 1997-1999. Arq Bras Oftalmol 2002;65;265-84.

\title{
VI SIMPÓSIO INTERNACIONAL DA SANTA CASA DE MISERICÓRDIA DE PORTO ALEGRE
}

\author{
11 e 12 de Julho de 2003 \\ AN FITEATRO HUGO GERDAU \\ SAN TA CASA DE PORTO ALEGRE - RS
}

INFORMAÇÕES: e-mail: eventos@santacasa.tche.br 